\title{
Maximum Principle and the Fourth Order Boundary Value Problem
}

\section{Yermachenko}

\section{Daugavpils University}

Parādes str. 1, Daugavpils, LV-5400, Latvia

E-mail: inara.jermacenko@du. $1 \mathrm{v}$

Received September 2, 2010; revised February 10, 2011; published online March 1, 2011

Abstract. This paper is devoted to the study the nonlinear boundary value problem (i) $x^{(4)}(t)+c^{2} x^{\prime \prime}(t)=f(t, x(t)),($ ii $) x(a)=A, x^{\prime}(a)=A_{1}, x(b)=B, x^{\prime}(b)=B_{1}$. We state a maximum principle related with the operator $u^{(4)}+c^{2} u^{\prime \prime}$ and apply it to prove the existence and approximation of solutions to the problem $(i),(i i)$, in presence of properly ordered lower and upper functions.

Keywords: Fourth order maximum principle, nonlinear boundary value problem, upper and lower functions.

AMS Subject Classification: 34A40; 34B15.

\section{Introduction}

Our motivation goes from the papers $[3,4]$, where equation

$$
x^{(4)}+c^{2} x^{\prime \prime}+e^{x}-1=0
$$

was considered. This equation comes from the theory of suspension bridges. The authors claim that for some value of $c$ there are multiple solutions.

We investigate the problem

$$
\begin{aligned}
& x^{(4)}(t)+c^{2} x^{\prime \prime}(t)=f(t, x(t)), \quad t \in I:=[a, b], \\
& x(a)=A, \quad x^{\prime}(a)=A_{1}, \quad x(b)=B, \quad x^{\prime}(b)=B_{1},
\end{aligned}
$$

where $A, A_{1}, B, B_{1} \in \mathbb{R}$. Our goal is to explore the method of lower and upper functions in order to prove the existence of a solution under suitable conditions.

It is well known for some kind of the second order boundary value problems (see $[7,10]$ ) that the existence of a lower function $\alpha$ and upper function $\beta$, such that $\alpha \leq \beta$, implies the existence of a solution of the considered problem. However the use of lower and upper functions in the fourth order boundary value problems is heavily dependent on the positiveness properties for the corresponding linear operators. Therefore we investigate a maximum principle for 
the operator

$$
(L u)(t):=u^{(4)}(t)+c^{2} u^{\prime \prime}(t)
$$

subject to the boundary conditions

$$
u(a)=u^{\prime}(a)=u(b)=u^{\prime}(b)=0
$$

and apply it to obtain existence theorems in the presence of properly ordered lower and upper functions.

Maximum principles are assertions which state that if some linear expression $(l u)(t)$ is of a definite sign for some nonconstant function $u(t)$ satisfying boundary conditions then $u(t)$ cannot attain its maximum or minimum values at an interior point. Maximum principles for the second order equations were considered by Protter and Weinberger [9] and for the fourth order equations in the papers $[1,2,5,8]$. Maximum principles have been successfully used to prove a solvability of boundary value problems [6].

\section{Maximum Principle}

Maximum principles for operators $u^{(4)}(t)$ and $u^{(4)}(t)+g(t) u^{\prime \prime \prime}(t)+h(t) u^{\prime \prime}(t)$ with the boundary conditions $u^{\prime}(a) \geq 0, u^{\prime}(b) \leq 0$ were formulated and proved in papers $[1,5]$ and $[2,8]$, respectively. The following theorems are valid.

Theorem 1 [[1, 5]]. Let $u \in C^{4}[a, b]$ satisfies the inequalities $u^{4}(t) \leq 0$, $t \in(a, b), \quad u^{\prime}(a) \leq 0, u^{\prime}(b) \geq 0$ and moreover attains its maximum value at $a$ point $t_{0} \in(a, b)$. Then $u$ is constant on $[a, b]$.

Theorem 2 [[2, 8]]. Let $u \in C^{4}(a, b) \cap C^{2}[a, b]$ satisfies the differential inequalities

$$
\begin{aligned}
& u^{4}(t)+g(t) u^{\prime \prime \prime}(t)+h(t) u^{\prime \prime}(t) \leq 0, \quad t \in(a, b), \\
& u^{\prime}(a) \leq 0, \quad u^{\prime}(b) \geq 0,
\end{aligned}
$$

where the functions $g$ and $h$ are bounded on every closed subinterval of $(a, b)$. If there exists a function $w \in C^{2}[a, b]$ such that

$$
w(t)<0, \quad t \in[a, b], \quad w^{\prime \prime}(t)+g(t) w^{\prime}(t)+h(t) w(t) \geq 0, \quad t \in(a, b),
$$

then $u$ cannot assume a maximum value at an interior point of $(a, b)$ unless $u$ is identically constant.

Remark 1. The condition of Theorem 2, that there exists a function $w(t)<0$ such that the inequality $w^{\prime \prime}(t)+g(t) w^{\prime}(t)+h(t) w(t) \geq 0$ is fulfilled, is a description of the linear differential expression in (2.1) (i.e., an additional assumption on functions $g$ and $h$ ). It is like to describe properties of $x^{\prime \prime}+k^{2} x$ depending on values of a parameter $k$ by requirement that there should exist a negative solution of $x^{\prime \prime}+k^{2} x \geq 0$ on a given interval $(a, b)$.

In this paper we will obtain a variant of Theorem 2 for the case of linear operator (1.4) with additional boundary conditions. Before doing that, we state the following lemma. 
Lemma 1. Let $w \in C^{2}[a, b]$ satisfies the differential inequality

$$
w^{\prime \prime}(t)+c^{2} w(t) \leq 0, \quad t \in(a, b)
$$

where $0<|c|(b-a)<\pi$, and moreover $w$ satisfies the boundary conditions $w(a) \geq 0, w(b) \geq 0$. Then for all $t \in[a, b], w(t) \geq 0$.

Proof. Consider the boundary value problem

$$
\begin{aligned}
& w^{\prime \prime}(t)+c^{2} w(t)=\varphi(t), \\
& w(a)=A, \quad w(b)=B,
\end{aligned}
$$

where $t \in I:=[a, b]$, a function $\varphi \in C[a, b]$ is non-positive, that is, for all $t \in[a, b], \varphi(t) \leq 0, A$ and $B$ are non-negative numbers, that is, $A \geq 0, B \geq 0$. The respective homogeneous problem

$$
\begin{aligned}
& w^{\prime \prime}(t)+c^{2} w(t)=0, \\
& w(a)=0, \quad w(b)=0
\end{aligned}
$$

is non-resonant if $|c|(b-a) \neq \pi n, n \in \mathbb{Z}$. Since $0<|c|(b-a)<\pi$ in a case under consideration, then the problem (2.4), (2.5) has only the trivial solution and therefore the problem $(2.2),(2.3)$ is solvable for any continuous function $\varphi(t)$. Notice that a solution $w(t)$ of the problem (2.2), (2.3) satisfies the inequalities mentioned in statement of Lemma 1. This solution can be written in the form

$$
w(t)=\frac{A \sin (|c|(b-t))+B \sin (|c|(t-a))}{\sin (|c|(b-a))}+\int_{a}^{b} G(t, s) \varphi(s) d s,
$$

where $G(t, s)$ is the Green function for the problem $(2.4),(2.5)$. It is given by

$$
G(t, s)= \begin{cases}\frac{\sin (|c|(t-b)) \sin (|c|(s-a))}{|c| \sin (|c|(b-a))}, & a \leq s \leq t \leq b, \\ \frac{\sin (|c|(s-b)) \sin (|c|(t-a))}{|c| \sin (|c|(b-a))}, & a \leq t<s \leq b .\end{cases}
$$

It follows from $0<|c|(b-a)<\pi$ that for all $(t, s) \in[a, b] \times[a, b], G(t, s) \leq 0$. Taking into account positiveness of $A, B$ and negativeness of function $\varphi$ we obtain that for all $t \in[a, b], w(t) \geq 0$. The proof is completed.

The maximum principle for the operator (1.4) can be formulated as follows:

Theorem 3. Let coefficient $c \in \mathbb{R}$ be such that

$$
0<|c|(b-a)<\pi
$$

and function $u \in C^{4}[a, b]$ satisfies the differential inequalities

$$
\begin{aligned}
& u^{(4)}(t)+c^{2} u^{\prime \prime}(t) \leq 0, \quad t \in(a, b) \\
& u(a) \leq 0, \quad u(b) \leq 0, \quad u^{\prime}(a) \leq 0, \quad u^{\prime}(b) \geq 0 .
\end{aligned}
$$

Then $u$ cannot assume a non-negative maximum value at an interior point of $(a, b)$ unless $u$ is identically zero. 
Proof. Suppose $u$ assumes a non-negative maximum value $M$ at some interior point $t_{0} \in(a, b), u\left(t_{0}\right)=M \geq 0$. Then $u^{\prime}\left(t_{0}\right)=0$ and $u^{\prime \prime}\left(t_{0}\right) \leq 0$. There exists a point $\xi \in\left[a, t_{0}\right)$ such that

$$
u(\xi) \leq 0, \quad u^{\prime}(\xi)=0, \quad u^{\prime \prime}(\xi) \geq 0 .
$$

Similarly there exists a point $\eta \in\left(t_{0}, b\right]$ such that

$$
u(\eta) \leq 0, \quad u^{\prime}(\eta)=0, \quad u^{\prime \prime}(\eta) \geq 0 .
$$

Denote $w(t)=u^{\prime \prime}(t)$ and consider a function $w$ in the interval $[\xi, \eta]$. We obtain that function $w$ satisfies the inequalities

$$
\begin{aligned}
& w^{\prime \prime}(t)+c^{2} w(t) \leq 0, \quad t \in[\xi, \eta], \\
& w(\xi) \geq 0, \quad w(\eta) \geq 0 .
\end{aligned}
$$

Therefore in accordance with Lemma 1 for all $t \in[\xi, \eta] w(t) \geq 0$. Since $w\left(t_{0}\right)=u^{\prime \prime}\left(t_{0}\right) \leq 0$ then $w \equiv 0$ in interval $[\xi, \eta]$. So $u^{\prime \prime} \equiv 0$ and therefore $u^{\prime} \equiv$ const in $[\xi, \eta]$. Since $u^{\prime}(\xi)=0$ and $u^{\prime}(\eta)=0$ then $u^{\prime} \equiv 0$ and $u \equiv$ const in $[\xi, \eta]$.

It follows from $u\left(t_{0}\right)=M \geq 0$ and $u(\xi) \leq 0, u(\eta) \leq 0$ that $u \equiv M=0$ in $[\xi, \eta]$. By continuity of $u$ a set of non-negative maximum points in $(a, b)$, i.e.

$$
K=\{t \in(a, b): u(t)=M=0\}
$$

is open relative to $(a, b)$, then we have $u \equiv 0$ in $[a, b]$.

Corollary 1. If a function $u \in C^{4}[a, b]$ satisfies the inequalities $(2.7),(2.8)$ and moreover the inequality $(2.6)$ holds, then $u(t) \leq 0$ in $[a, b]$.

Remark 2. Assumption (2.6) in Theorem 3 and Corollary 1 is essential. Indeed, the nonconstant function $u(t)=\cos (c t)$ satisfies the inequalities $(2.7),(2.8)$ in the interval $[-\pi / c, \pi / c]$, but it assumes a positive maximum at the interior point $t_{0}=0$. A contradiction with the assertion of the Theorem 3 follows from the fact that inequality (2.6) is not satisfied.

Remark 3. The results of Theorem 3 (as well as Corollary 1) continue to hold if all the inequalities involving a function $u$ are reversed, provided the words "non-negative maximum" are replaced by the words "non-positive minimum". Thus the minimum principle may be formulated for the linear operator (1.4).

Although the maximum principle stated above seems interesting in itself, our main purpose is to apply it to prove the existence theorem for the nonlinear fourth order boundary value problem (1.2), (1.3).

\section{Lower and Upper Functions}

Consider the boundary value problem (1.2), (1.3), where $f \in C(I \times \mathbb{R}, \mathbb{R})$ and a coefficient $c \in \mathbb{R}$ satisfies (2.6). The respective homogeneous problem

$$
\begin{aligned}
& x^{(4)}(t)+c^{2} x^{\prime \prime}(t)=0, \\
& x(a)=0, \quad x^{\prime}(a)=0, \quad x(b)=0, \quad x^{\prime}(b)=0
\end{aligned}
$$


is non-resonant, that is, it has only the trivial solution, if and only if the following condition is satisfied:

$$
2|c| \sin \frac{|c|(b-a)}{2}\left(\frac{|c|(b-a)}{2} \cos \frac{|c|(b-a)}{2}-\sin \frac{|c|(b-a)}{2}\right) \neq 0 .
$$

So if the inequality (2.6) holds then problem (3.1), (3.2) has only the trivial solution.

In order to prove the solvability of the nonlinear boundary value problem under consideration we shall modify the given original equation (1.2) to a quasilinear one using a function $\delta$, where

$$
\delta(\lambda, \mu, \nu)= \begin{cases}\lambda, & \mu<\lambda \\ \mu, & \lambda \leq \mu \leq \nu \\ \nu, & \mu>\nu\end{cases}
$$

Theorem 4. Let in the boundary value problem (1.2), (1.3) $f \in C(I \times \mathbb{R}, \mathbb{R})$, $c \in \mathbb{R}$ satisfies (2.6), $A, A_{1}, B, B_{1} \in \mathbb{R}$. Suppose that there exist functions $\alpha, \beta \in C^{(4)}[a, b]$ such that

(1) $\alpha(t) \leq \beta(t), t \in[a, b]$;

(2) $\alpha^{(4)}(t)+c^{2} \alpha^{\prime \prime}(t) \leq f(t, \delta(\alpha, x, \beta)), t \in[a, b], x \in \mathbb{R}$,

$$
\beta^{(4)}(t)+c^{2} \beta^{\prime \prime}(t) \geq f(t, \delta(\alpha, x, \beta)), t \in[a, b], x \in \mathbb{R} ;
$$

(3) $\alpha(a) \leq A, \alpha(b) \leq B, \alpha^{\prime}(a) \leq A_{1}, \alpha^{\prime}(b) \geq B_{1}$,

$$
\beta(a) \geq A, \beta(b) \geq B, \beta^{\prime}(a) \geq A_{1}, \beta^{\prime}(b) \leq B_{1} .
$$

Then there exists a solution $x(t)$ of the problem (1.2), (1.3) such that $\alpha(t) \leq$ $x(t) \leq \beta(t), t \in[a, b]$.

Functions $\alpha(t), \beta(t)$ are called lower and upper function respectively.

Proof. Consider the modified equation

$$
x^{(4)}(t)+c^{2} x^{\prime \prime}(t)=f(t, \delta(\alpha, x, \beta)),
$$

where

$$
f(t, \delta(\alpha, x, \beta))= \begin{cases}f(t, \alpha(t)), & x(t)<\alpha(t), \\ f(t, x(t)), & \alpha(t) \leq x(t) \leq \beta(t), \\ f(t, \beta(t)), & x(t)>\beta(t) .\end{cases}
$$

This modification of $f$ is widely used in the theory of nonlinear boundary value problems [10]. Obviously the function $f(t, \delta(\alpha, x, \beta))$ is continuous in $I \times \mathbb{R}$ and bounded; so the equation (3.3) is a quasi-linear one. Since the respective homogeneous problem (3.1), (3.2) for $c$ satisfying (2.6) has only the trivial solution, thus in accordance with the Conti's theorem there exists a solution $x(t)$ of the quasi-linear problem (3.3), (1.3).

Denote $u(t)=x(t)-\beta(t)$. It follows from (3.3) and (2) that

$$
\begin{aligned}
u^{(4)}+c^{2} u^{\prime \prime} & =x^{(4)}+c^{2} x^{\prime \prime}-\beta^{(4)}-c^{2} \beta^{\prime \prime} \\
& \leq f(t, \delta(\alpha, x, \beta))-f(t, \delta(\alpha, x, \beta))=0,
\end{aligned}
$$


then from (1.3) and (3) it follows that $u(a) \leq 0, u(b) \leq 0, u^{\prime}(a) \leq 0, u^{\prime}(b) \geq$ 0 . Thus $u$ satisfies the inequalities $(2.7),(2.8)$, therefore in accordance with Theorem 3 and Corollary $1 u(t) \leq 0$, that is, $x(t) \leq \beta(t), t \in[a, b]$.

Similarly we prove that $\alpha(t) \leq x(t), t \in[a, b]$. Then the lower and upper bounds $\alpha(t) \leq x(t) \leq \beta(t), t \in[a, b]$ are valid. This solution $x(t)$ solves the original problem (1.2), (1.3) too.

Remark 4. If a function $f(t, x)$ is monotone in $x$ then the conditions (2) of Theorem 4 can be simplified. If $f(t, x)$ is increasing in $x$, then it suffices to require that

$$
\begin{aligned}
& \beta^{(4)}(t)+c^{2} \beta^{\prime \prime} \geq f(t, \beta(t)), \quad t \in[a, b] ; \\
& \alpha^{(4)}(t)+c^{2} \alpha^{\prime \prime} \leq f(t, \alpha(t)), \quad t \in[a, b] .
\end{aligned}
$$

If $f(t, x)$ is decreasing in $x$, then it suffices to require that

$$
\begin{array}{ll}
\beta^{(4)}(t)+c^{2} \beta^{\prime \prime} \geq f(t, \alpha(t)), & t \in[a, b] ; \\
\alpha^{(4)}(t)+c^{2} \alpha^{\prime \prime} \leq f(t, \beta(t)), & t \in[a, b] .
\end{array}
$$

\section{Application}

Example 1. Consider the problem

$$
\begin{aligned}
& x^{(4)}+x^{\prime \prime}=x^{3}+0.1, \\
& x(-a)=0=x(a), \quad x^{\prime}(-a)=0=x^{\prime}(a), \quad 0<a<\frac{\pi}{2} .
\end{aligned}
$$

The function $f(x):=x^{3}+0.1$ is increasing in $x$, so we try to find an upper function $\beta(t)$ such that

$$
\begin{aligned}
& \beta^{(4)}(t)+\beta^{\prime \prime}(t) \geq \beta^{3}(t)+0.1, \quad t \in[-a, a] \\
& \beta(-a) \geq 0, \quad \beta(a) \geq 0, \quad \beta^{\prime}(-a) \geq 0, \quad \beta^{\prime}(a) \leq 0
\end{aligned}
$$

and a lower function $\alpha(t)$ such that

$$
\begin{aligned}
& \alpha^{(4)}(t)+\alpha^{\prime \prime}(t) \leq \alpha^{3}(t)+0.1, \quad t \in[-a, a] \\
& \alpha(-a) \leq 0, \quad \alpha(a) \leq 0, \quad \alpha^{\prime}(-a) \leq 0, \quad \alpha^{\prime}(a) \geq 0 .
\end{aligned}
$$

We can choose $\alpha(t) \equiv 0$ and $\beta(t)=\gamma \cos \left(\left(\frac{\pi}{2 a}-\varepsilon\right) t\right)$, where $\gamma>0$ and $\varepsilon \geq 0$. Then the inequalities (4.3) are fulfilled and (4.2) takes the form

$$
\begin{aligned}
\gamma\left(\frac{\pi}{2 a}-\varepsilon\right)^{2}\left(\left(\frac{\pi}{2 a}-\varepsilon\right)^{2}-1\right) & \cos \left(\left(\frac{\pi}{2 a}-\varepsilon\right) t\right) \\
& \geq \gamma^{3} \cos ^{3}\left(\left(\frac{\pi}{2 a}-\varepsilon\right) t\right)+0.1
\end{aligned}
$$

If $a=1$ then the inequality (4.4) holds, for instance, for $\gamma=0.4, \varepsilon=0.2$, therefore in this case the problem (4.1) has a solution $x(t)$ such that $0 \leq$ $x(t) \leq 0.4 \cos \left(\left(\frac{\pi}{2}-0.2\right) t\right), t \in[-1,1]$. We have computed this solution, but it is difficult to show on the same figure the graphs of upper and lower functions and a graph of $x(t)$, because two lines $(\alpha(t)$ and $x(t))$ almost coincide. 
Example 2. Consider the problem

$$
\begin{aligned}
& x^{(4)}+2 x^{\prime \prime}=-x^{3}+0.1, \\
& x(-1)=0=x(1), \quad x^{\prime}(-1)=0=x^{\prime}(1) .
\end{aligned}
$$

The function $f(x):=-x^{3}+0.1$ is decreasing in $x$, so we try to find the upper $\beta(t)$ and lower $\alpha(t)$ functions such that

$$
\begin{aligned}
& \beta^{(4)}+4 \beta^{\prime \prime} \geq-\alpha^{3}+0.1, \quad t \in[-1,1], \\
& \beta(-1) \geq 0, \quad \beta(1) \geq 0, \quad \beta^{\prime}(-1) \geq 0, \quad \beta^{\prime}(1) \leq 0 ; \\
& \alpha^{(4)}+4 \alpha^{\prime \prime} \leq-\beta^{3}+0.1, \quad t \in[-1,1], \\
& \alpha(-1) \leq 0, \quad \alpha(1) \leq 0, \quad \alpha^{\prime}(-1) \leq 0, \quad \alpha^{\prime}(1) \geq 0 .
\end{aligned}
$$

We can find these functions in the form

$$
\beta(t)=\gamma\left(t^{2}-1\right)\left(t^{2}-(1+\varepsilon)^{2}\right), \quad \alpha(t)=-\gamma\left(t^{2}-1\right)\left(t^{2}-(1+\varepsilon)^{2}\right),
$$

where $\gamma>0, \varepsilon>0$. Then the inequalities (4.7), (4.9) will be fulfilled. Both inequalities (4.6) and (4.8) hold if the following inequality is satisfied:

$$
24 \gamma-4 \gamma\left(2+2 \varepsilon+\varepsilon^{2}-6 t^{2}\right) \geq \gamma^{3}\left(t^{2}-1\right)^{3}\left(t^{2}-(1+\varepsilon)^{2}\right)^{3}+0.1
$$

The inequality (4.10) holds if $\gamma=0.01, \varepsilon=0.1$. Therefore upper and lower functions exist and the problem (4.5) is solvable.

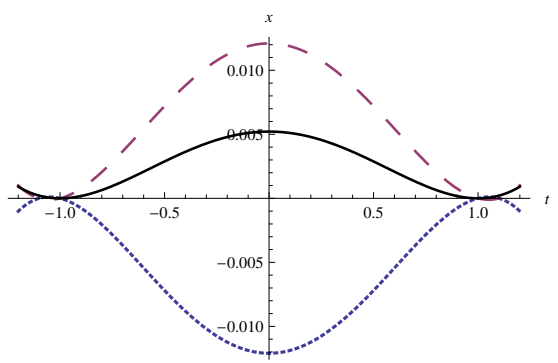

Figure 1. The solution of problem (4.5) and corresponding upper and lower functions.

The solid line in Fig. 1 indicates a solution of problem (4.5) and dashed lines present the corresponding upper and lower functions.

In papers $[3,4]$ the nonlinear beam equation (1.1) was considered and the existence of at least 36 travelling wave solutions for $c=1.3$ was proved. We consider this equation together with different boundary conditions and prove the solvability of the respective boundary value problems applying the method of upper and lower functions.

Example 3. Consider the problem

$$
\begin{aligned}
& x^{(4)}+1.3^{2} x^{\prime \prime}=1-e^{x}, \\
& x(-1)=1=x(1), \quad x^{\prime}(-1)=0=x^{\prime}(1) .
\end{aligned}
$$


The function $f(x):=1-e^{x}$ is decreasing in $x$, so we try to find the upper $\beta(t)$ and lower $\alpha(t)$ functions such that

$$
\begin{aligned}
& \beta^{(4)}+1.3^{2} \beta^{\prime \prime} \geq 1-e^{\alpha}, \quad t \in[-1,1], \\
& \beta(-1) \geq 1, \quad \beta(1) \geq 1, \quad \beta^{\prime}(-1) \geq 0, \quad \beta^{\prime}(1) \leq 0 ; \\
& \alpha^{(4)}+1.3^{2} \alpha^{\prime \prime} \leq 1-e^{\beta}, \quad t \in[-1,1], \\
& \alpha(-1) \leq 1, \quad \alpha(1) \leq 1, \quad \alpha^{\prime}(-1) \leq 0, \quad \alpha^{\prime}(1) \geq 0 .
\end{aligned}
$$

We try to find them in the form

$$
\begin{aligned}
& \beta(t)=\gamma_{\beta}\left(t^{2}-1\right)\left(t^{2}-\left(1+\varepsilon_{\beta}\right)^{2}\right)+1+\delta_{\beta}, \\
& \alpha(t)=-\gamma_{\alpha}\left(t^{2}-1\right)\left(t^{2}-\left(1+\varepsilon_{\alpha}\right)^{2}\right)+1-\delta_{\alpha},
\end{aligned}
$$

where $\gamma_{\beta}, \gamma_{\alpha}, \varepsilon_{\beta}, \varepsilon_{\alpha}$ are positive and $\delta_{\beta}, \delta_{\alpha}$ are non-negative. In this case the inequalities (4.13), (4.15) are fulfilled and the inequalities (4.12), (4.14) take the following forms respectively:

$$
\begin{aligned}
& 2 \gamma_{\beta}\left(12+1.69\left(6 t^{2}-\left(2+2 \varepsilon_{\beta}+\varepsilon_{\beta}^{2}\right)\right)\right) \geq 1-e^{\alpha(t)}, \\
& -2 \gamma_{\alpha}\left(12+1.69\left(6 t^{2}-\left(2+2 \varepsilon_{\alpha}+\varepsilon_{\alpha}^{2}\right)\right)\right) \leq 1-e^{\beta(t)} .
\end{aligned}
$$

Notice that (4.16) is satisfied for all $t \in[-1,1]$ if a minimum value of the left-hand side expression in this interval will be greater than or equal to a maximum value of the right-hand side expression in this interval. The lefthand side expression is a quadratic function of $t$ and it assumes its minimum value at the point $t=0$. The right-hand side expression assumes its maximum value at the same point $t=0$. For $t=0$ the inequality (4.16) takes form

$$
2 \gamma_{\beta}\left(12-1.69\left(2+2 \varepsilon_{\beta}+\varepsilon_{\beta}^{2}\right)\right) \geq 1-e^{-\gamma_{\alpha}\left(1+\varepsilon_{\alpha}\right)^{2}+1-\delta_{\alpha}} .
$$

(4.16) holds for all $t \in[-1,1]$ if (4.18) is fulfilled. Similarly obtain that (4.17) holds for all $t \in[-1,1]$ if the following inequality is satisfied

$$
2 \gamma_{\alpha}\left(1.69\left(2+2 \varepsilon_{\alpha}+\varepsilon_{\alpha}^{2}\right)-12\right) \leq 1-e^{\gamma_{\beta}\left(1+\varepsilon_{\beta}\right)^{2}+1+\delta_{\beta}} .
$$

If $\delta_{\alpha}=\delta_{\beta}=0, \varepsilon_{\alpha}=\varepsilon_{\beta}=0.1, \gamma_{\alpha}=\gamma_{\beta}=0.14$ then both inequalities (4.18) and (4.19) are fulfilled, thus in this case there exist the upper and lower functions and the problem (4.11) is solvable.

Fig. 2(a) illustrates a solution $x(t)$ of the nonlinear problem (4.11) in the interval $[-1,1]$. In Fig. 2(b) this solution is shown in solid line and dashed lines present the corresponding upper and lower functions. The same solution $x(t)$ but in the interval $[-15,15]$ is depicted in Fig. 2(c).

Consider the same nonlinear equation subjected fo slightly modified boundary conditions

$$
\begin{aligned}
& x^{(4)}+1.3^{2} x^{\prime \prime}=1-e^{x}, \\
& x(-1)=-1=x(1), \quad x^{\prime}(-1)=0=x^{\prime}(1) .
\end{aligned}
$$



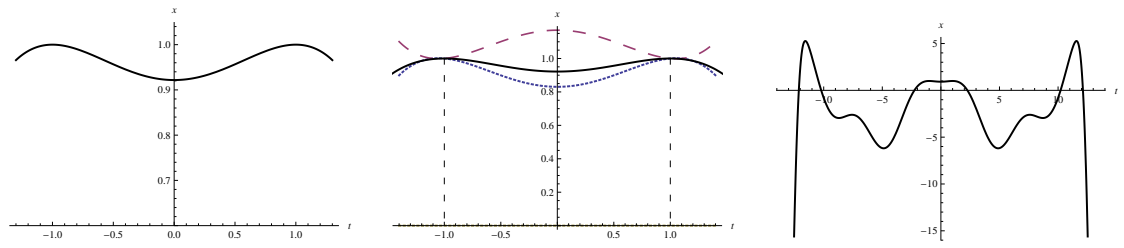

Figure 2. Solution of the problem (4.11).

In this case we find the upper and lower functions in the form

$$
\begin{aligned}
& \beta(t)=\gamma_{\beta}\left(t^{2}-1\right)\left(t^{2}-\left(1+\varepsilon_{\beta}\right)^{2}\right)-1+\delta_{\beta}, \\
& \alpha(t)=-\gamma_{\alpha}\left(t^{2}-1\right)\left(t^{2}-\left(1+\varepsilon_{\alpha}\right)^{2}\right)-1-\delta_{\alpha} .
\end{aligned}
$$

Then (4.12) and (4.14) hold for all $t \in[-1,1]$ if the following inequalities are fulfilled

$$
\begin{aligned}
& 2 \gamma_{\beta}\left(12-1.69\left(2+2 \varepsilon_{\beta}+\varepsilon_{\beta}^{2}\right)\right) \geq 1-e^{-\gamma_{\alpha}\left(1+\varepsilon_{\alpha}\right)^{2}-1-\delta_{\alpha}}, \\
& 2 \gamma_{\alpha}\left(1.69\left(2+2 \varepsilon_{\alpha}+\varepsilon_{\alpha}^{2}\right)-12\right) \leq 1-e^{\gamma_{\beta}\left(1+\varepsilon_{\beta}\right)^{2}-1+\delta_{\beta}} .
\end{aligned}
$$

For instance, these inequalities (4.21), (4.22) are satisfied if $\delta_{\alpha}=\delta_{\beta}=0$, $\varepsilon_{\alpha}=\varepsilon_{\beta}=0.1, \gamma_{\alpha}=0.02$ and $\gamma_{\beta}=0.04$, thus for such values of mentioned constants there exist the upper and lower functions and the problem (4.20) is solvable.
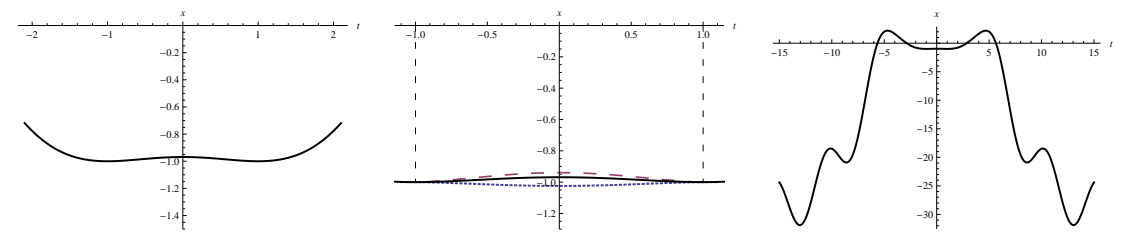

Figure 3. Solution of the problem (4.20).

A solution $x(t)$ of the nonlinear problem (4.20) in the interval $[-1,1]$ is shown in Fig. 3(a). Fig. 3(b) illustrates this solution in solid line and dashed lines present the corresponding upper and lower functions. The same solution $x(t)$ but in the interval $[-15,15]$ is depicted in Fig. 3(c).

Comparing Fig. 2(c) and Fig. 3(c)can be seen that we have proved the existence of another solution of the nonlinear beam equation.

\section{Conclusion}

A maximum principle for the fourth order linear operator $(L u)(t):=u^{(4)}(t)+$ $c^{2} u^{\prime \prime}(t)$ is formulated and proved. The existence theorem (Theorem 4) for twopoint nonlinear boundary value problem (1.2), (1.3) is proved in presence of 
properly ordered lower and upper functions. In Examples 1 and 2 methods of construction of lower and upper functions are discussed.

Theorem 4 was used to prove the existence of multiple solutions of the beam equation (Example 3). As a by product we have proven the existence of solutions, which satisfy the given two-point boundary conditions.

\section{References}

[1] R.P. Agarwal. Boundary Value Problems for Higher Order Differential Equations. World Scientific Publ. Co Pte Ltd., 1986.

[2] J. Bochenek. On a maximum principle for fourth order ordinary differential inequalities. Universitatis Jagellonicae Acta Math., 27:163-168, 1988.

[3] B. Breuer, J. Horák, P.J. McKenna and M. Plum. A computer-assisted existence and multiplicity proof for travelling waves in a nonlinearly supported beam. $J$. Differential Equations, 224:60-97, 2006. Doi:10.1016/j.jde.2005.07.016.

[4] B. Breuer, J. Horák, P.J. McKenna and M. Plum. Travelling waves in a nonlinearly supported beam: A computer-assisted existence and multiplicity proof. In AIP Conf. Proceed., Int. Conf. on Num. Analysis and App. Math., pp. 869-872, 2009 .

[5] S.N. Chow, D.R. Dunninger and A. Lasota. A maximum principle for fourth order ordinary differential equations. J. Differential Equations, 14:101-105, 1973. Doi:10.1016/0022-0396(73)90079-X.

[6] R. Čiegis, G. Jankevičiūtè and O. Suboč. Numerical simulation of the heat conduction in composite materials. Mathematical Modelling and Analysis, 15(1):922, 2010. Doi:10.3846/1392-6292.2010.15.9-22.

[7] C.De Coster and P. Habets. Two-Point Boundary Value Problems: Lower and Upper Solutions. Elsevier, 2006.

[8] D.R. Dunninger. Maximum principle for fourth order ordinary differential inequalities. J. Math. Anal. Appl., 82:399-405, 1981. Doi:10.1016/0022-247X(81)90205-5.

[9] M.H. Protter and H.F. Weinberger. Maximum Principles in Differential Equations. Prentice-Hall, Englewood Cliffs, NJ, 1967.

[10] N.I. Vasilyev and Yu.A. Klokov. Foundations of Theory of Boundary Value Problems for Ordinary Differential Equations. Zinatne, Riga, 1978. (in Russian) 